\title{
Evidências da origem e ascensão dos dinossauros sauropodomorfos preservadas em leitos fossilíferos do Triássico do Sul do Brasil
}

\author{
Evidence OF the ORIGIN AND RISE OF SAURopodomorph dinosaurs preserved in Triassic beds from Southern Brazil \\ Rodrigo Temp Müllere ${ }^{1}$, Maurício Silva Garcia², Sérgio Dias-da-Silva² \\ 1 - Centro Apolo Pesq. Paleont. da Quarta Colônia, Univ. Fed. Santa Maria, São João do Polêsine, Brasil. \\ 2 - Laboratório de Paleobiodiversidade Tríssica, Departamento de Ecologia e Evolução, Univ. Fed. Santa Maria, Santa Maria, Brasil.

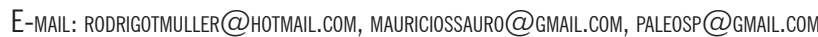

\begin{abstract}
Although the sauropodomorph dinosaur group is widely known due to the giant sauropods, their origin is still poorly understood due to the scarcity of complete skeletons and precise dating. However, new findings and dating performed in Triassic rocks from Brazil have brought forth valuable information regarding this matter. The combination of new data and more advanced investigation techniques (e.g. CT scans) have enabled the establishment of macroevolutionary patterns that guided the initial evolution of the group. Through discoveries made in Triassic beds of Rio Grande do Sul state, we can understand in more detail how sauropodomorphs went from small and not abundant animals to the largest animals that ever walked the Earth.
\end{abstract}

Resumo: Embora o grupo dos dinossauros sauropodomorfos seja amplamente conhecido em virtude dos gigantescos saurópodes, sua origem ainda é pouco compreendida em virtude da escassez de esqueletos completos ou datações precisas. Entretanto, novos achados e datações realizadas em rochas Triássicas do Brasil têm trazido à tona informações valiosas no que tange essa questão. A combinação desses novos dados com técnicas mais avançadas de investigação (e.g. tomografias computadorizadas) vem possibilitando o estabelecimento dos padrões macroevolutivos que direcionaram a evolução inicial do grupo. Por meio das descobertas realizadas nos leitos Triássicos do Rio Grande do Sul, podemos entender de forma mais refinada de que forma os sauropodomorfos passaram de animais pequenos e pouco abundantes aos maiores animais que já andaram sobre a Terra.
Citation/Citação: Müller, R. T., Garcia, M. S., \& Silva, S. D. (2020). Evidências da origem e ascensão dos dinossauros sauropodomorfos preservadas em leitos fossilíferos do Triássico do Sul do Brasil. Terræ Didatica, 16, 1-13, e020013. doi:10.20396/td.v16i0.8657367

Keywords: Dinosauria. Evolution. Mesozoic Era. Saurischia. Sauropodomorpha.

Palavras-chave: Dinosauria. Evolução. Era Mesozoica. Saurischia. Sauropodomorpha.

\section{Manuscript/Manuscrito:}

Received/Recebido: 20/01/2020

Revised/Corrigido: 20/02/2020

Accepted/Aceito: 05/04/2020

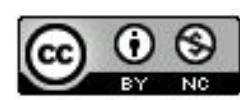

\section{Introdução}

O grupo dos dinossauros sauropodomorfos é um dos mais conhecidos, até mesmo por aqueles que não tem familiaridade com a área, afinal, o grupo inclui os maiores animais terrestres que já existiram, os quais são facilmente reconhecidos pelo seu pescoço imenso. Porém, estes representantes são membros de um grupo mais específico chamado de Sauropoda. No entanto, antes dos primeiros dinossauros sauropodomorfos serem capazes de se elevar sobre suas patas posteriores e esticar seu longo pescoço em direção a um ramo de vegetação que lhes servisse de alimento, uma longa jornada foi trilhada por seus precursores avemetatarsalianos (linhagem que inclui os dinossauros, mas também outras formas mais 'primitivas') durante o Triássico Inicial [entre 252 e 247 milhões de anos atrás (Ma)] e Médio (247-237 Ma). Abordagens filogenéticas indicam que a linhagem aviana (i.e. dinossauros e formas aparentadas, pterossauros e afanossauros) de arcossauros separou-se da linhagem crocodiliana durante o Início do Triássico, há cerca de $252 \mathrm{Ma}$ (Nesbitt et al., 2017). Enquanto os pseudossúquios (arcossauros mais relacionados aos crocodilos do que às aves) alcançaram grande sucesso evolutivo durante o Triássico Médio e início do Triássico Superior (237-201 Ma), a linhagem avemetatarsaliana passou a exercer dominância ecológica sobre os ambientes continentais somente a partir da metade do Triássico Superior (Brusatte et al., 2008).

O clado mais basal de Avemetatarsalia é chamado de Aphanosauria (Nesbitt et al., 2017) e inclui algumas formas quadrupedes de aparência um tanto enigmática, já que esse clado retém características presentes em Pseudosuchia, como a morfologia geral do calcanhar e as proporções 
dos membros. Até o momento, o registro fóssil do grupo estende-se do início do Triássico Médio até o início do Triássico Superior, com representantes tanto no paleocontinente Gondwana (incluindo o Rio Grande do Sul) como no de Laurásia (Nesbitt et al., 2017). Os afanossauros correspondem ao grupo irmão de Ornithodira, o clado que engloba pterossauros, dinossauros e as formas aparentadas (Fig. 1). Enquanto Pterosauria não possui até o momento registros em rochas do Triássico Inferior e Médio, seu grupo irmão Dinosauromorpha (Benton, 1985) é representado por vários esqueletos fósseis a partir do Triássico Médio (Nesbitt et al., 2010), além de possíveis registros icnológicos (pegadas) em rochas do Triássico Inferior (Brusatte et al., 2011). As evidências filogenéticas apontam até o momento para a existência de três principais clados de dinossauromorfos. O mais basal, Lagerpetidae (Arcucci, 1986), inclui cerca de meia dúzia de espécies supostamente cursoriais (animais corredores com membros delgados, tal como a espécie encontrada no Rio Grande do Sul: Ixalerpeton polesinensis), as quais se estendem temporalmente ao longo de quase todo o Triássico Superior (e.g., Irmis et al., 2007, Cabreira et al., 2016, Martínez et al., 2016, Müller et al., 2018a, Garcia et al., 2019a). A biologia desse clado ainda é pouco compreendida em virtude da incompletude dos espécimes já descobertos. Por exemplo, o único material craniano atribuído a Lagerpetidae refere-se a uma caixa craniana (Cabreira et al., 2016), de modo que até o momento nenhum elemento ósseo contendo dentes foi registrado, dificultando a investigação dos seus hábitos alimentares. Além disso, com exceção de um espécime descoberto na Argentina (Martínez et al., 2016), todos outros espécimes indicam um tamanho corpóreo pequeno (não mais que 1 metro de comprimento). Os fósseis mais antigos de lagerpetídeos são registrados em rochas carnianas (237-227 Ma) da América do Sul (Romer, 1971, Cabreira et al., 2016, Garcia et al., 2019a), ao passo que formas de idade noriana (227-208 Ma) são também registradas na América do Norte (Irmis et al., 2007, Sarigül, 2016). Embora pouco se conheça ainda sobre esse enigmático grupo, estudos investigando séries ontogenéticas de lagerpetídeos (e.g. Nesbitt et al., 2009) têm servido como base para melhorar o entendimento a respeito da biologia do desenvolvimento de dinossauromorfos.

Com pouco mais de dez espécies válidas, o segundo ramo que compõe o clado dos dinossauromorfos é chamado de Silesauridae (Langer et al., 2010, Nesbitt et al., 2010). Tal ramo está mais próximo daquele que inclui os dinossauros, formando junto com eles e algumas poucas formas de posicionamento geralmente flutuante, o clado Dinosauriformes (Novas, 1992). Todavia, ainda existe uma linha de evidências que pode sugerir um relacionamento ainda mais próximo entre silessaurídeos e dinossauros, onde os primeiros na verdade estariam inseridos em Ornithischia, um dos três principais grupos de dinossauros. Sendo assim, silessaurídeos poderiam na verdade ter sido dinossauros (e.g. Langer \& Ferigolo, 2013, Cabreira et al., 2016) ao invés de grupo-irmão dos mesmos (e.g. Nesbitt et al., 2010). Temporalmente, os silessaurídeos estendem-se do Anisiano (247-242 Ma) até próximo do final do Noriano (Nesbitt et al., 2010), o que faz destes os dinossauromorfos com os registros corpóreos mais antigos conhecidos. De fato, o registro fóssil deste grupo é muito mais abrangente em comparação com o registro de afa-

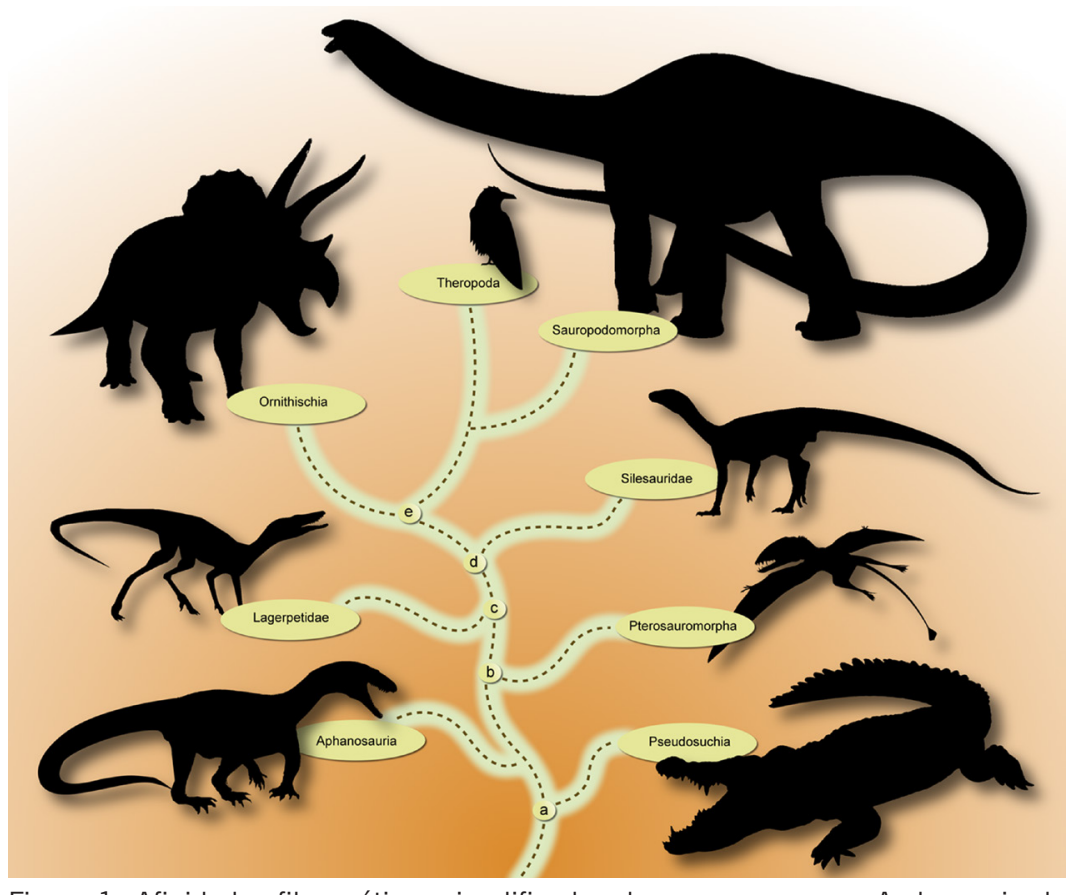

Figura 1. Afinidades filogenéticas simplificadas dos arcossauros. a, Archosauria; b, Ornithodira; c, Dinosauromorpha; d, Dinosauriformes; e, Dinosauria 
nossauros e lagerpetídeos, o que ajuda a entender diferentes aspectos da biologia do grupo, como, por exemplo, seu hábito alimentar, até o momento sugerido como herbívoro ou onívoro com base nos dentes e morfologia do crânio e mandíbula (Nesbitt et al., 2010, Cabreira et al., 2016). Além disso, os membros anteriores bastante alongados sugerem que este grupo foi formado por animais quadrúpedes, contrastando com o padrão bípede dos primeiros dinossauros. A maioria das formas não chegava a ultrapassar 1,5 metros de comprimento (e.g. Ferigolo \& Langer, 2007), entretanto um possível espécime de silessaurídeo escavado em rochas da Tanzânia pode ter chegado a medir quase 3 metros de comprimento (Barrett et al., 2015). Assim como os lagerpetídeos, seu padrão de distribuição geográfica aponta para uma origem gondwanica, posteriormente alcançando ampla distribuição geográfica também no Laurásia (Dzik, 2003). Tal como todos outros grupos de dinossauromorfos não-dinossaurianos, os silessaurídeos tornaram-se extintos pouco antes de alcançar o final do Triássico (201 Ma), porém o grupo coexistiu com seus parentes próximos dinossaurianos por cerca de 20 milhões de anos (Irmis et al., 2007, Garcia et al., 2019a).

Por fim, o terceiro e esmagadoramente mais diverso ramo de dinossauromorfos, Dinosauria (Owen, 1842), é agrupado em três principais clados menos inclusivos: Ornithischia, Theropoda e Sauropodomorpha. Por muito tempo, os dois últimos foram considerados filogeneticamente mais próximos, formando o grande clado Saurischia (Seeley, 1887). No entanto, essa afinidade tem sido desafiada a partir de uma nova, porém não amplamente adotada, hipótese que sugere maior afinidade entre terópodes e ornitísquios (Baron et al., 2017). De qualquer forma, pode-se observar características distintas em cada um dos três clados, de modo que entender a evolução de cada um deles corresponde a um desafio à parte. Somam-se ainda a essa difícil tarefa a presença de algumas formas de posicionamento consideravelmente instável, como os herrerassaurídeos, já classificados tanto como parte dos terópodes, como também como saurísquios basais à dicotomia Theropoda/Sauropodomorpha (veja Langer et al., 2010, Cabreira et al., 2016). Em relação ao registro fóssil de dinossauros, as formas inequívocas mais antigas são reportadas a partir de rochas de idade Carniana da América do Sul, além de registros fragmentários da África e Índia (Ezcurra, 2012). Todavia, as três principais linhagens não são registradas de maneira sincrônica em rochas dessa idade. Com relação aos estudos dos dinossauros, os ornitísquios foram por muito tempo somente representados por Pisanosaurus mertii (Casamiquela, 1967), da Formação Ischigualasto da Argentina. Entretanto, uma reavaliação filogenética apontou possíveis afinidades deste táxon com silessaurídeos (Agnolín \& Rozadilla, 2017), fazendo assim com que ornitísquios não mais passassem ser representados em estratos do Carniano. $\mathrm{Na}$ verdade, esse posicionamento coloca o início do Período Jurássico (201-145 Ma) como o registro mais antigo de ornitísquios, dado que nenhum outro espécime inequívoco é reconhecido para o Triássico até o momento (Baron, 2017). Entretanto cabe-se ressaltar que, se a hipótese que reconhece silessaurídeos como ornitísquios for considerada, o grupo passa a possuir um registro bastante completo durante o Triássico (Cabreira et al., 2016).

De forma semelhante ao exposto acima, se reconhecermos os herrerassaurídeos como terópodes, o registro desses últimos aumenta drasticamente para o Carniano. Contudo, terópodes registrados nesses estratos e que não façam supostamente parte do grupo dos herrerassaurídeos são extremamente raros e geralmente questionáveis. Um dos mais completos espécimes corresponde a Eodromaeus murphi (Martínez et al., 2011), da Formação Ischigualasto da Argentina. Porém a inclusão desse táxon em Theropoda foi recentemente desafiada por meio de uma hipótese que sugere um posicionamento basal à dicotomia Theropoda/ Sauropodomorpha (Cabreira et al., 2016). Outro táxon com potenciais afinidades com Theropoda é Nhandumirim waldsangae (Marsola et al., 2019), da Formação Santa Maria (= porção inferior da Sequência Candelária) do Brasil. Entretanto um estudo filogenético mais abrangente indicou que este táxon poderia na verdade fazer parte de Sauropodomorpha (Pacheco et al., 2019). Diferentemente do registro de Ornithischia, os terópodes passam a ser registrados mais frequentemente em rochas de idade Noriana e Raetiana (208-201 Ma) (Pinheiro, 2016, Ezcurra, 2017, Martínez \& Apaldetti, 2017).

Finalmente, o registro de sauropodomorfos é, de longe, o mais abundante em comparação com outros clados dinossaurianos durante o Triássico. O mesmo é verdade para os estratos Carnianos, nos quais os sauropodomorfos alcançam a maior diversidade, com três espécies da Formação Ischigualasto da Argentina e quatro para a Formação Santa Maria do Brasil. Embora possam apresentar

\begin{tabular}{c|c|c|c|c|c}
\hline C Terrae Didat. & Campinas, SP & v.16 & $1-13$ & $\mathrm{e} 020013$ & 2020 \\
\hline
\end{tabular}


maior diversidade taxonômica, a abundancia desses animais ainda é pequena quando comparada com outros táxons recuperados nessas mesmas unidades, tais como cinodontes (grupo de sinápsidos que origina os mamíferos) ou pseudossúquios (Martínez et al., 2013), fato que se torna oposto quando examinados os registros a partir do final do Noriano (Müller et al., 2017). De qualquer modo, independentemente da linhagem a qual pertençam, todos os principais registros de dinossauros de idade Carniana apontam para um plano corpóreo bípede, com mãos livres, um pescoço não muito alongado e dentição não completamente especializada para uma dieta herbívora (Cabreira et al., 2016, Müller et al., 2018b), indicando possíveis traços do plano corporal ancestral do grupo.

\section{A ascensão do "Império Prossaurópoda"}

Enquanto rochas de idade Carniana preservaram poucos registros fósseis de sauropodomorfos, camadas de idades mais jovens apresentam registros muito abundantes desse clado em grande parte do mundo. Essa mudança abrupta no conteúdo fossilífero referente a sauropodomorfos parece refletir um importante câmbio biótico, no qual estes passam de formas pequenas e raras para os vertebrados terrestres mais abundantes em seus ecossistemas. O evento é conhecido como o "Império Prosauropoda" (Benton, 1983) e marca o primeiro momento em que os dinossauros efetivamente passam a exercer domínio sobre os ecossistemas terrestres. O principal fator intrínseco ligado a essa mudança parece estar relacionado à aquisição de uma dieta herbívora. Enquanto formas mais basais apresentam características que indicam hábito alimentar faunívoro (Cabreira et al., 2016, Bronzati et al., 2017), as formas sucessivamente mais derivadas registradas próximo ao final do Triássico possuem diversas adaptações relacionadas a uma dieta herbívora. Somando-se ainda a essa tendência, outras mudanças ocorreram durante esse período de transição, como por exemplo o aumento do tamanho corpóreo e o alongamento do pescoço, ambos permitindo o forrageio de vegetação mais alta do que qualquer outro grupo de vertebrados era capaz de alcançar nessa época (Parrish, 1998). Desta forma, parece que a adoção de um novo estilo de dieta em combinação com tais características, tenha sido um dos principais responsáveis pela ascensão do grupo. Porém, o tempo e modo em que essas características se estabeleceram ainda é mal compreendido, dada a escassez de fósseis de sauropodomorfos do exato intervalo de tempo de transição, o qual deve ter ocorrido possivelmente durante o início e a metade do Noriano (Müller et al., 2017). Todavia, novos achados realizados em rochas do Triássico do Sul do Brasil têm lançado luz sobre a questão (Müller et al., 2018c).

Diversas controvérsias filogenéticas também afetam as tentativas de estabelecer padrões mais complexos relacionados ao grupo. O mais conhecido desafio filogenético que envolve os sauropodomorfos está ligado à disputa entre dois cenários: (1) os "prossaurópodes" (sauropodomorfos de médio a grande porte, bípedes, com pescoço longo e crânio pequeno) comporiam um arranjo monofilético (Fig. 2A) (Upchurch et al., 2007) e (2) seriam parafiléticos (Fig. 2B) (Yates 2007). O aumento de espécimes que tem ocorrido ao longo dos últimos anos parece dar mais suporte ao último cenário, indicando que os "prossaurópodes" formariam uma série com poucos táxons sucessivamente parafiléticos em relação aos saurópodes propriamente ditos. Neste ponto, outro desafio entra em cena. Este diz respeito à falta de consenso do que exatamente constitui um "verdadeiro" saurópode, muitas vezes relacionado à postura quadrúpede (McPhee \& Choiniere, 2017). Evolução convergente também já foi apontada como responsável por produzir distintos sinais filogenéticos entre o crânio e o pós-crânio de alguns sauropodomorfos (Apaldetti et al., 2014). Finalmente, outra dificuldade que assola o estudo do grupo envolve a flutuabilidade de certos táxons, especialmente as formas mais basais e algumas formas norte americanas (Rowe et al., 2010, Sertich \& Loewen, 2010). Além de reduzir a robustez de ramos específicos, essa condição também interfere nas tentativas de estabelecer padrões biogeográficos e evolutivos.

Ainda que muitas controvérsias pairem sobre as afinidades internas de Sauropodomorpha, esforços de coleta ao redor do mundo tem contribuído na construção de um cenário promissor. Já é possível entender tanto a forma do esqueleto, como aspectos biológicos das formas mais basais do grupo (Cabreira et al., 2016). Técnicas computacionais de reconstrução de tecidos moles (e.g. encéfalo) têm possibilitado acesso a informações antes desconhecidas (e.g. Bronzati et al., 2017) e estudos paleohistológicos e ontogenéticos vem revelando padrões biológicos intrigantes, como por exemplo, grandes mudanças anatômicas em certas regiões do esqueleto (Apaldetti et al., 2018, Müller et al., 
A
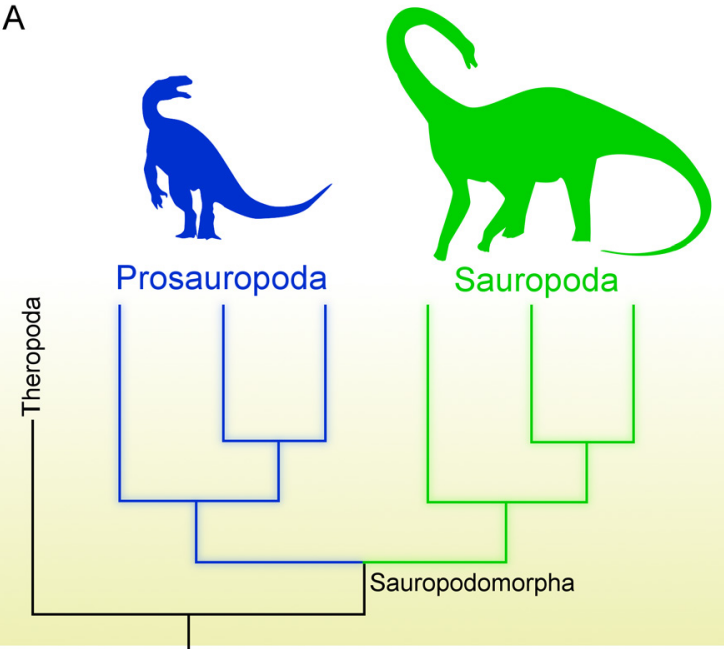

B

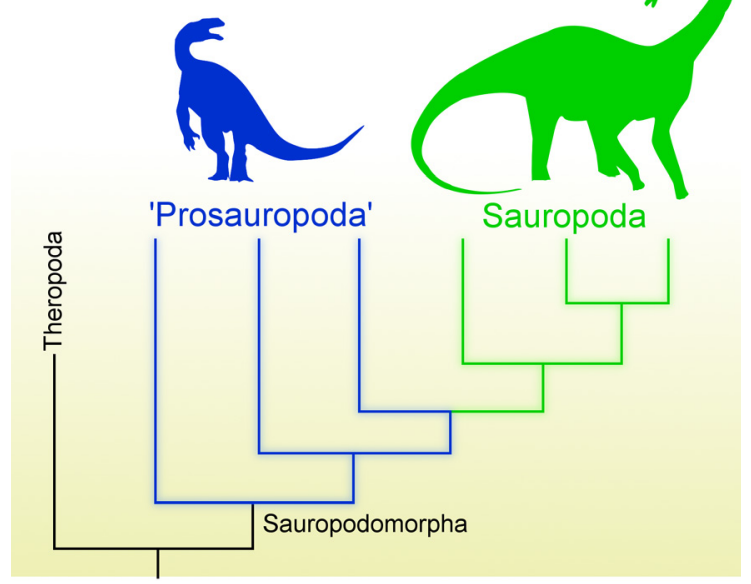

Figura 2. Principais hipóteses filogenéticas acerca das afinidades dos 'prossaurópodes'. A) Prosauropoda monofilético, B) 'Prosauropoda' parafilético

2018d, Garcia et al., 2019b). Entretanto, ainda resta muito a ser feito para que seja possível contar a história evolutiva dos sauropodomorfos com maior acuidade e riqueza de detalhes.

\section{Definindo um sauropodomorfo}

Ao se deparar com um esqueleto fóssil de um animal gigante, quadrupede, de pescoço incrivelmente longo, cabeça pequena e dentição herbívora, não irão restar dúvidas, trata-se de um dinossauro sauropodomorfo. Mas e se esse esqueleto for de um dinossauro pequeno, bípede, cabeça proporcionalmente um pouco maior, com pescoço curto e dentes em forma de punhal? Seria um equívoco reconhecê-lo como um sauropodomorfo? Esse é um desafio que vem sendo enfrentado por aqueles que estudam as formas mais basais de sauropodo- morfos. Por muitos anos, o pequeno saurísquio Eoraptor lunensis (Sereno et al., 1993), da Formação Ischigualasto da Argentina, foi reconhecido como um terópode, dado que apresentava todas essas características mencionadas acima e que, até então, eram reconhecidas somente em terópodes. Porém, a descoberta de um outro esqueleto que recebeu o nome de Eodromaeus murphi (Martínez et al., 2011), trouxe alguns questionamentos à tona. Esse esqueleto também compartilhava todas as características mencionadas anteriormente e ainda possuía outras feições comuns aos terópodes, enquanto que estas eram ausentes em Eoraptor. Tal fato levou os pesquisadores a examinar com mais detalhes a anatomia de Eoraptor, o que por fim revelou vários traços compartilhados com os sauropodomorfos, como por exemplo, a abertura ampla da narina, o processo ventral do esquamosal delgado e o primeiro dente do dentário localizado posteriormente à extremidade anterior da mandíbula (Martínez et al., 2011). Assim, quase duas décadas após a descrição original, Eoraptor passou a ser aninhado junto aos outros sauropodomorfos. Esse novo arranjo mudou em vários aspectos o modo de entender a origem do grupo, afinal, Eoraptor não parecia portar uma dentição típica de um animal herbívoro ou onívoro (dentes recurvados posteriormente com serrilhas finas formando um ângulo reto em relação ao eixo do dente). Foi então que, em 2016, um novo sauropodomorfo de idade Carniana, da Formação Santa Maria (correspondente à porção inferior da Sequência Candelária) foi descrito. O táxon recebeu o nome de Buriolestes schultzi (Cabreira et al., 2016) e foi apontado como membro mais basal de Sauropodomorpha, de modo que apresentava um aspecto ainda menos parecido com as formas típicas de grupo, especialmente em relação à forma de sua dentição, claramente de um animal faunívoro, onde a coroa dentária é notoriamente recurvada posteriormente e munida de serrilhas finas que formam um ângulo reto em relação ao eixo principal do dente. Apesar de bastante completo, o esqueleto que corresponde ao holótipo de Buriolestes carrega poucas sinapomorfias que servem para suportar seu parentesco com outros sauropodomorfos, sendo a extremidade anterior do dentário ventralmente direcionada uma das principais características (Cabreira et al., 2016). Esse é, de fato, um cenário plausível quando se está lidando com as formas mais basais de um clado, especialmente quando se conhece pouco acerca da morfologia ancestral e das formas basais dos gru-

\begin{tabular}{c|c|c|c|c|c|}
\hline C) Terrae Didat. & Campinas, SP & v.16 & $1-13$ & $\mathrm{e} 020013$ & 2020 \\
\hline \multicolumn{4}{|c}{5}
\end{tabular}


pos relacionados. Como resultado, a retenção de características plesiomórficas (i.e. característica primitiva) pode ser erroneamente interpretada como características compartilhadas por clados próximos. Por exemplo, a dentição zifodonte (especializada para faunivoria) de Buriolestes aparentemente corresponde a condição plesiomórfica de dinossauros, no entanto, poderia ser equivocadamente identificada como uma sinapomorfia compartilhada com terópodes, uma vez que formas mais derivadas de sauropodomorfos não possuem tal padrão dentário e só recentemente este foi reconhecido também em outras formas basais, como Eoraptor e Saturnalia tupiniquim (Martínez et al., 2011, Cabreira et al., 2016, Bronzati et al., 2017, Müller et al., 2018c).

Deste modo, ao se deparar com formas supostamente próximas da origem de um clado, em se tratando de Sauropodomorpha, torna-se particularmente difícil classificar seus integrantes com base no uso de "caracteres-chave" (e.g. narinas externas amplas, processo ventral do esquamosal delgado, digito I da mão hipertrofiado), especialmente se o espécime em questão for composto por poucos elementos ósseos. Sendo assim, identificar um sauropodomorfo pode ser mais razoável por meio de sua inclusão por meio de uma abordagem filogenética, seguindo a definição em vigência proposta para esse clado. A definição filogenética para Sauropodomorpha mais aceita atualmente foi proposta por Taylor et al. (2010) como "o clado mais inclusivo contendo Saltasaurus loricatus mas não Tyrannosaurus rex". Essa definição engloba também as formas mais basais, ao contrário da definição mais antiga proposta por Salgado et al. (1997) que excluía essas formas, uma vez que foi construída utilizando o grupo "Prosauropoda", que atualmente é considerado sinônimo de Plateosauridae de acordo com a definição proposta para "Prosauropoda" por Galton \& Upchurch (2007): "todos os táxons mais proximamente relacionados a Plateosaurus do que a Sauropoda". Uma outra definição mais recentemente proposta por Baron et al. (2017) considera Sauropodomorpha como "o clado mais inclusivo que contém Diplodocus carnegii, mas não Triceratops horridus, Passer domesticus ou Herrerasaurus ischigualastensis. Embora essa definição tenha sido proposta para um contexto onde sauropodomorfos estariam menos relacionados com terópodes (i.e. terópodes como grupo irmão de ornitísquios = Ornithoscelida), ela também serve para definir o grupo em esquemas filogenéticos tradicionais (i.e. Saurischia composto por terópodes e sauropodomorfos).

\section{Sauropodomorfos Triássicos do Rio Grande do Sul}

Sauropodomorfos são registrados em rochas triássicas da Sequência Candelária (sensu Horn et al., 2014) no Rio Grande do Sul (Fig. 3), a qual tem ocorrência em municípios como Candelária, Agudo, Faxinal do Soturno, São João do Polêsine, Santa Maria e São Martinho da Serra. Essa Sequência faz parte da Supersequência Santa Maria, a qual abrange um intervalo temporal que vai do Triássico Médio até próximo do final do Triássico (Zerfass et al., 2003). A Sequência Candelária, por sua vez, possui um alcance temporal que se inicia aproximadamente na metade do Carniano (cerca de $233 \mathrm{Ma}$ ) e vai até pouco antes da metade do Noriano (cerca de $225 \mathrm{Ma}$ ) (Langer et al., 2018), e esta unidade vem produzindo os registros mais antigos de sauropodomorfos, permitindo o rastreio da evolução inicial do grupo. De fato, já são conhecidas quatro espécies provenientes da porção inferior dessa Sequência, além de mais duas espécies da porção superior. A porção inferior da Sequência Candelária envolve a parte superior da Formação Santa Maria, que teve seu primeiro sauropodomorfo descrito em 1999, Saturnalia tupiniquim (Langer et al., 1999) (Fig. 4A). A espécie, oriunda do município de Santa Maria, é conhecida por uma série de esqueletos parciais escavados na localidade tipo. Mais de dez anos depois, foi descrito Pampadromaeus barberenai (Cabreira et al., 2011) (Fig. 4B), proveniente de um sítio localizado no município de Agudo. Além do holótipo, compreendendo um esqueleto incompleto, são também conhecidos alguns espécimes compostos por elementos isolados, todos da localidade tipo (Müller et al., 2018d). A espécie seguinte recebeu o nome de Buriolestes schultzi (Cabreira et al., 2016) (Fig. 4C) e foi descrita a partir de um esqueleto parcial e alguns elementos isolados de outros indivíduos descobertos no município de São João do Polêsine. Finalmente, a espécie mais recentemente apresentada, Bagualosaurus agudoensis (Pretto et al., 2018) (Fig. 5A), foi descrita a partir de um esqueleto parcial coletado na mesma localidade que revelou Pampadromaeus barberenai. Além das quatro espécies mencionadas, vale ressaltar que Nhandumirim waldsangae (Marsola et al., 2019), que foi inicialmente descrito como um potencial terópode, pode na verdade ser um sauropodomorfo de acordo com um estudo mais recente de Pacheco et al. (2019). Essa espécie é conhecida por um único esqueleto fragmentário de um indivíduo juvenil 
coletado no município de Santa Maria, na mesma localidade fossilífera que produziu os esqueletos de Saturnalia tupiniquim.

Dado o exposto acima, pode-se observar um súbito aumento taxonômico de sauropodomorfos de idade Carniana para o Brasil nos últimos anos, possivelmente relacionado à ampliação numérica de equipes realizando trabalho de prospecção em rochas triássicas do Rio Grande do Sul, e também pelo

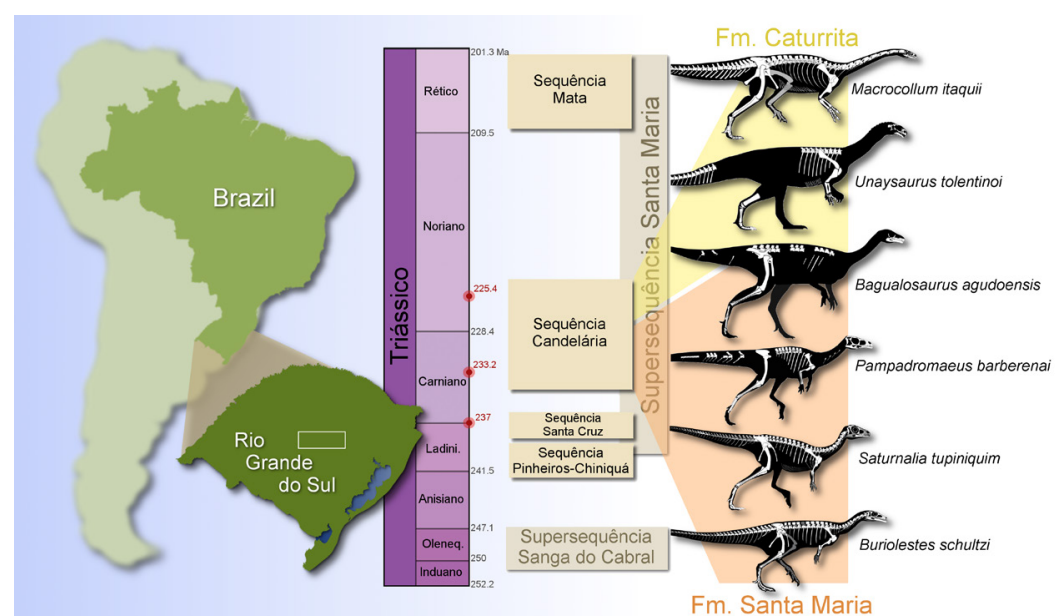

Figura 3. Localização geográfica e cronoestratigráfica dos registros de sauropodomorfos para o Triássico do Brasil, onde as idades em destaque correspondem a datações absolutas aumento na frequência de expedições de prospecção e coleta na região. Porém, segundo Müller \& Garcia (2019), essa alta diversidade de sauropodomorfos já durante o início da evolução do grupo poderia ser explicada por três hipóteses principais. A primeira delas sugere que na verdade os sauropodomorfos considerados de idade Carniana não tenham sido todos coevos. De fato, é possível que alguns estratos que fazem parte da Zona de Associação (camada caracterizada pela associação de certos grupos taxonômicos) de Hyperodapedon do Brasil sejam de idades diferentes, o que faria com que pelo menos Bagualosaurus agudoensis e Pampadromaeus barberenai fossem um pouco mais jovens do que Saturnalia tupiniquim e Buriolestes schultzi. De acordo, uma divisão similar é reconhecida também para os estratos argentinos (Martínez et al., 2013). A segunda hipótese levantada por Müller \& Garcia (2019) questiona a validade taxonômica de alguns sauropodomorfos em virtude de vieses ontogenéticos, uma vez que se sabe que a variação morfológica resultante de processos ontogenéticos é muito elevada em dinossauros basais e grupos relacionados (e.g. Griffin \& Nesbitt, 2016, Müller et al., 2018d, Garcia et al., 2019b). Assim, táxons considerados como duas ou mais espécies poderiam na verdade fazer parte da gama morfológica de uma única espécie durante seus estágios de desenvolvimento. Por fim, a terceira hipótese explica a diversidade de sauropodomorfos em um mesmo intervalo temporal pela ocupação de diferentes nichos ecológicos. Realmen- te, a dentição dos sauropodomorfos considerados de idade Carniana é bastante variável, sendo algumas espécies claramente faunívoras (e.g. Buriolestes schultzi, Saturnalia tupiniquim), ao passo que outras já apresentam características dentárias que estariam relacionadas a uma dieta onívora (Bagualosaurus agudoensis, Pampadromaeus barberenai). Neste cenário, diferentes espécies de sauropodomorfos poderiam coexistir, porém provavelmente não iriam competir avidamente por recursos (Müller \& Garcia 2019).

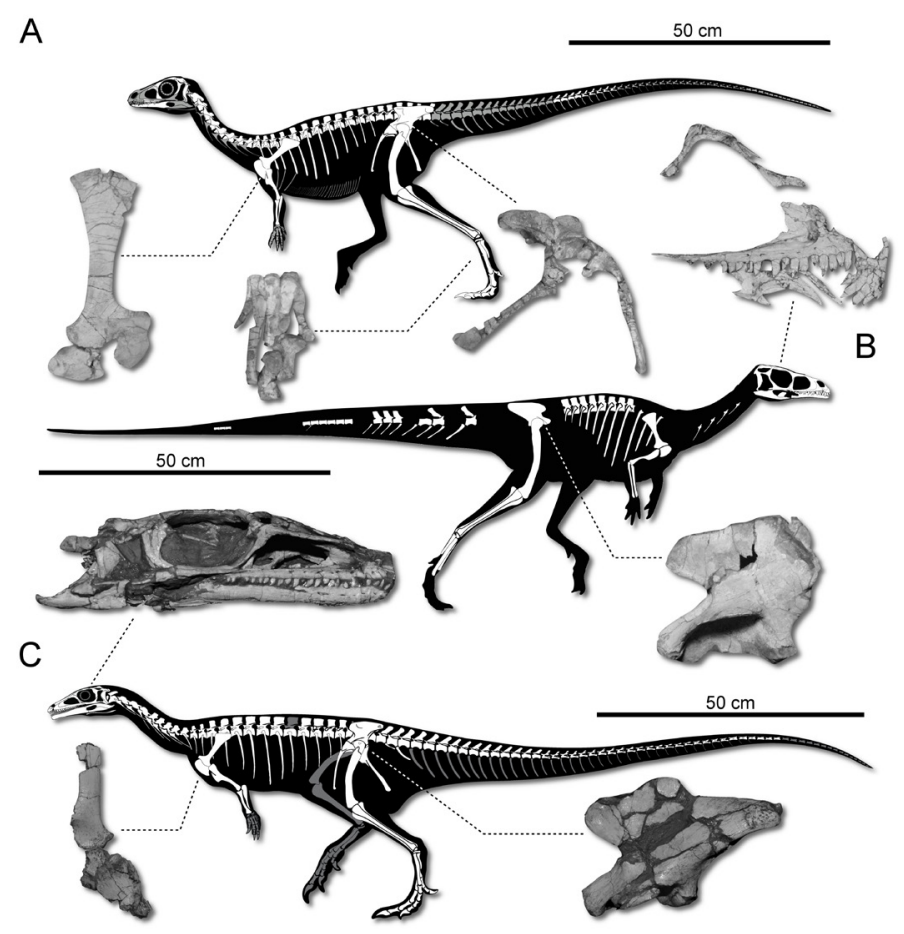

Figura 4. Sauropodomorfos do Triássico do Rio Grande do Sul (parte 1). A) Saturnalia tupiniquim, B) Pampadromaeus barberenai, C) Buriolestes schultzi

\begin{tabular}{c|c|c|c|c|c}
\hline (C) Terrae Didat. & Campinas, SP & v.16 & $1-13$ & e020013 & 2020 \\
\hline
\end{tabular}


De qualquer maneira, esse acréscimo amostral de sauropodomorfos carnianos não apenas tem expandido a diversidade do grupo, mas também incrementado a qualidade de seu registro. De fato, nunca antes tantos espécimes (muitos ainda em fase de preparação e estudo) oriundos desses estratos haviam sido escavados como nos últimos anos. Por conta disso, pode-se esperar que ao longo dos próximos anos, muitos trabalhos de pesquisa sejam realizados com enfoque em sauropodomorfos carnianos do Rio Grande do Sul. Por outro lado, a diversidade taxonômica desse clado a partir da porção superior da Sequência Candelária (correspondente à Formação Caturrita), não havia aumentado desde 2004, com a descrição de Unaysaurus tolentinoi (Leal et al., 2004) (Fig. 5B), escavado no município de São Martinho da Serra. Além de representar a única espécie inequívoca de Sauropodomorpha para esses estratos até pouco tempo atrás, também é conhecida por um único esqueleto incompleto. No entanto, vale ressaltar que esse não foi o único registro para esta unidade. De fato, até o momento já foram reportados vários espécimes (e.g. Bittencourt et al., 2012, 2013, Müller et al., 2015) atribuídos a Sauropodomorpha, no entanto, seu estado em maioria fragmentário ou a incompleto, torna a identificação em um grupo específico (i.e. menos inclusivo) bastante incerta. Entretanto, isso não é regra para todos os espécimes, sendo alguns deles muito bem preservados e completos. Este é o caso dos espécimes coletados no município de Agudo que serviram de base para elencar o táxon Macrocollum itaquii (Müller et al., 2018c) (Fig. 5C), o sauropodomorfo mais antigo de pescoço longo já descoberto. Além de praticamente completos (faltando apenas alguns ossos da caixa craniana), os esqueletos de Macrocollum itaquii foram preservados em um paleoambiente que permitiu que fossem fossilizados intimamente associados (i.e. esqueletos lado a lado ou mesmo se sobrepondo) e com similar grau de articulação, indicando possível habito gregário durante a evolução inicial dos sauropodomorfos (Müller et al., 2018c). Tais achados também tornam a porção superior da Sequência Candelária bastante promissora no que se refere ao estudo dos sauropodomorfos. Por fim, cabe salientar ainda que o dinossauro Guaibasaurus candelariensis (Bonaparte et al., 1999), registrado tanto no município de Candelária, como também em Faxinal do Soturno, já foi considerado por alguns autores como um membro de Sauropodomorpha (e.g. Novas et al., 2011). Por outro lado, há também estudos que defendem

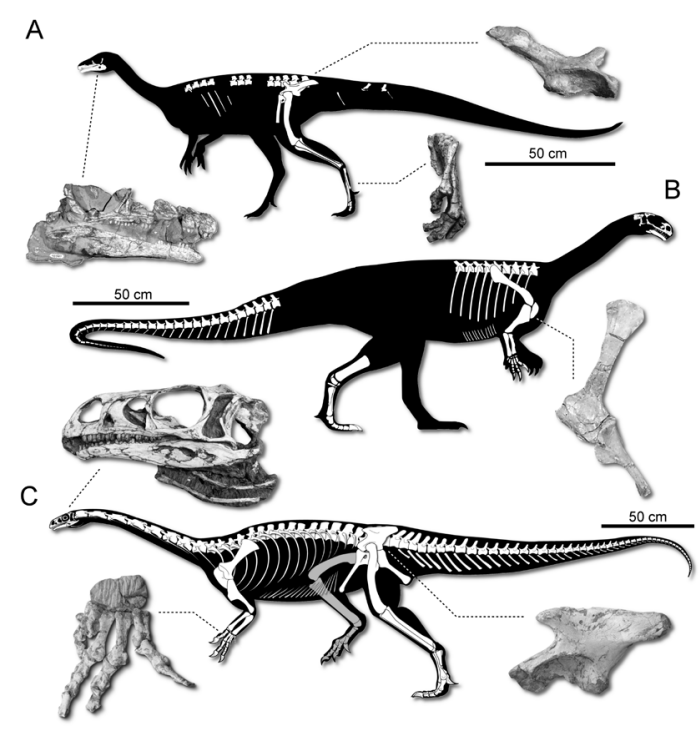

Figura 5. Sauropodomorfos do Triássico do Rio Grande do Sul (parte 2). A) Bagualosaurus agudoensis, B) Unaysaurus tolentinoi, C) Macrocollum itaquii

o posicionamento como membro de Theropoda (e.g. Langer et al., 2011) ou como um saurísquio basal a dicotomia Theropoda/Sauropodomorpha (Cabreira et al., 2016). Em parte, a dificuldade em reconhecer as afinidades filogenéticas deste táxon está relacionada com a falta de um crânio ou pescoço entre os espécimes conhecidos e com a preservação precária de alguns elementos de seu esqueleto (e.g. membros com extremidades articulares danificadas). Deste modo, o posicionamento filogenético deste dinossauro continua sendo um desafio e ainda requer espécimes mais completos para que se chegue em algum consenso.

Já em relação as afinidades filogenéticas dos sauropodomorfos do Rio Grande do Sul (Fig. 6), se observa certa concordância quanto ao posicionamento da maioria dos táxons. Na verdade, existem algumas divergências quanto as afinidades internas de alguns grupos menos inclusivos ou quanto ao posicionamento de táxons-chave. Não obstante, a maioria dos estudos concorda que Buriolestes schultzi (Cabreira et al., 2016, Müller et al., 2018b, Pretto et al., 2018, Bronzati et al., 2019) seria, ou o membro mais basal do grupo, ou um dos membros mais basais (Müller et al., 2018b, Bronzati et al., 2019, Langer et al., 2019). Já Saturnalia tupiniquim e Pampadromaeus barberenai vêm sendo recuperados como membros de Saturnaliinae (Müller et al., 2018c, Langer et al., 2019, Müller, 2019), embora $P$. barberenai já tenha sido também aninhado com Panphagia protos (Bronzati et al., 2019,, Müller et

\begin{tabular}{c|c|c|c|c|c}
\hline C Terrae Didat. & Campinas, SP & v.16 & $1-13$ & $\mathrm{e} 020013$ & 2020 \\
\hline
\end{tabular}




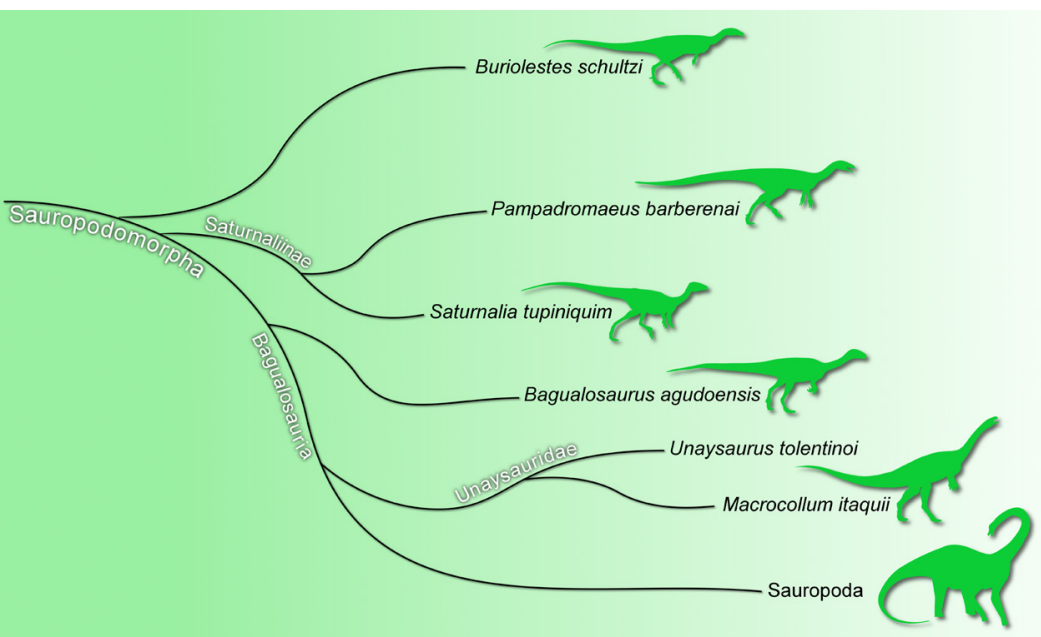

Figura 6. Afinidades filogenéticas simplificadas dos sauropodomorfos do Rio Grande do Sul (baseada na topologia de Müller et al., 2018c) al., 2011, Langer et al., 2018) e técnicas mais sofisticadas de investigação anatômica (e.g. tomografias computadorizadas) têm lançado luz sobre a origem e a irradiação inicial dos sauropodomorfos na América do Sul. A combinação destas linhas de investigação nos permite reconstruir com maior rigor as etapas envolvidas durante os primeiros milhões de anos de evolução destes animais (Fig. 7). Sabe-se, por exemplo, que as formas mais antigas de sauropodomorfos al., 2018b), em um clado irmão do nodo que une Saturnaliinae e Bagualosauria. Este último clado tem Bagualosaurus agudoensis como membro mais basal, de modo que este táxon seria mais relacionado aos sauropodomorfos de idade Noriana do que aqueles de idade Carniana (Pretto et al., 2018, Müller et al., 2018c, Bronzati et al., 2019, Langer et al., 2019, Müller, 2019). De fato, a posição filogenética de $B$. agudoensis condiz com seu tamanho e presença de certas características morfológicas, como um crânio proporcionalmente reduzido, ao passo que outras feições (e.g. presença da plataforma trocantérica no fêmur) remetem ele a condição usual de formas Carnianas.

Já os sauropodomorfos de idade Noriana seriam mais derivados quando comparados as formas de idade Carniana, o que é completamente plausível. Unaysaurus tolentinoi foi por muitos anos recuperado como um Plateosauridae, sendo táxon-irmão de Plateosaurus (Leal et al., 2004). Porém, uma análise abrangente levou $U$. tolentinoi a ser recuperado como membro de Unaysauridae, junto com Macrocollum itaquii (Müller et al., 2018c), onde este clado seria grupo-irmão de Plateosauria. No entanto, um estudo mais recente e ainda mais abrangente apontou uma hipótese alternativa, onde Unaysauridae poderia ser o grupo mais basal de Massopoda (Müller, 2019). Neste cenário os sauropodomorfos de idade Noriana do Rio Grande do Sul seriam mais derivados do que Plateosauridae.

\section{Considerações finais}

Os esforços de coleta dos últimos anos em associação com novas datações (e.g. Martínez et são oriundas de rochas de aproximadamente 233 milhões de anos (Langer et al., 2018). Essas formas são pequenas, faunívoras e bípedes, como pode ser observado pelo esqueleto fóssil de Buriolestes schult$z i$, por exemplo. No entanto, algumas delas, como o Saturnalia tupiniquim ou Bagualosaurus agudoensis, já apresentam uma redução no tamanho do crânio em relação ao tamanho corporal (Pretto et al., 2018, Bronzati et al., 2019), uma característica que viria a se tornar comum em todos os sauropodomorfos à partir do Noriano. Além disso, nota-se também o aumento gradual do tamanho corpóreo, onde Bagualosaurus agudoensis teria um comprimento femoral de cerca de 21,5 centímetros (Pretto et al., 2018), ao passo que Macrocollum itaquii, coletado em rochas de cerca de 225 milhões de anos, teria um comprimento femoral de cerca de 33,4 centímetros (Müller et al., 2018b). Esse aumento corpóreo também acompanha a mudança nas proporções entre tíbia e fêmur, onde formas mais basais, como Buriolestes schultzi, teriam a tíbia mais longa do que o fêmur, característica tipicamente cursorial, ao passo que em Macrocollum itaquii a tíbia é mais curta do que o fêmur. Por fim, uma das principais mudanças no plano corpóreo envolve o alongamento das vértebras cervicais, resultando em um pescoço até duas vezes mais longo nas formas do Noriano quando comparadas com àquelas do Carniano (Müller et al., 2018c).

Todas essas alterações parecem acompanhar a mudança na dentição, a qual passa a acumular traços relacionados a uma dieta herbívora (Müller et al., 2018c). De fato, um pescoço longo certamente ajudou os sauropodomorfos do Noriano a praticar forrageio de vegetação mais elevado, a qual outros

\begin{tabular}{c|c|c|c|c|c|}
\hline (C) Terrae Didat. & Campinas, SP & v.16 & $1-13$ & $\mathrm{e} 020013$ & 2020 \\
\hline
\end{tabular}




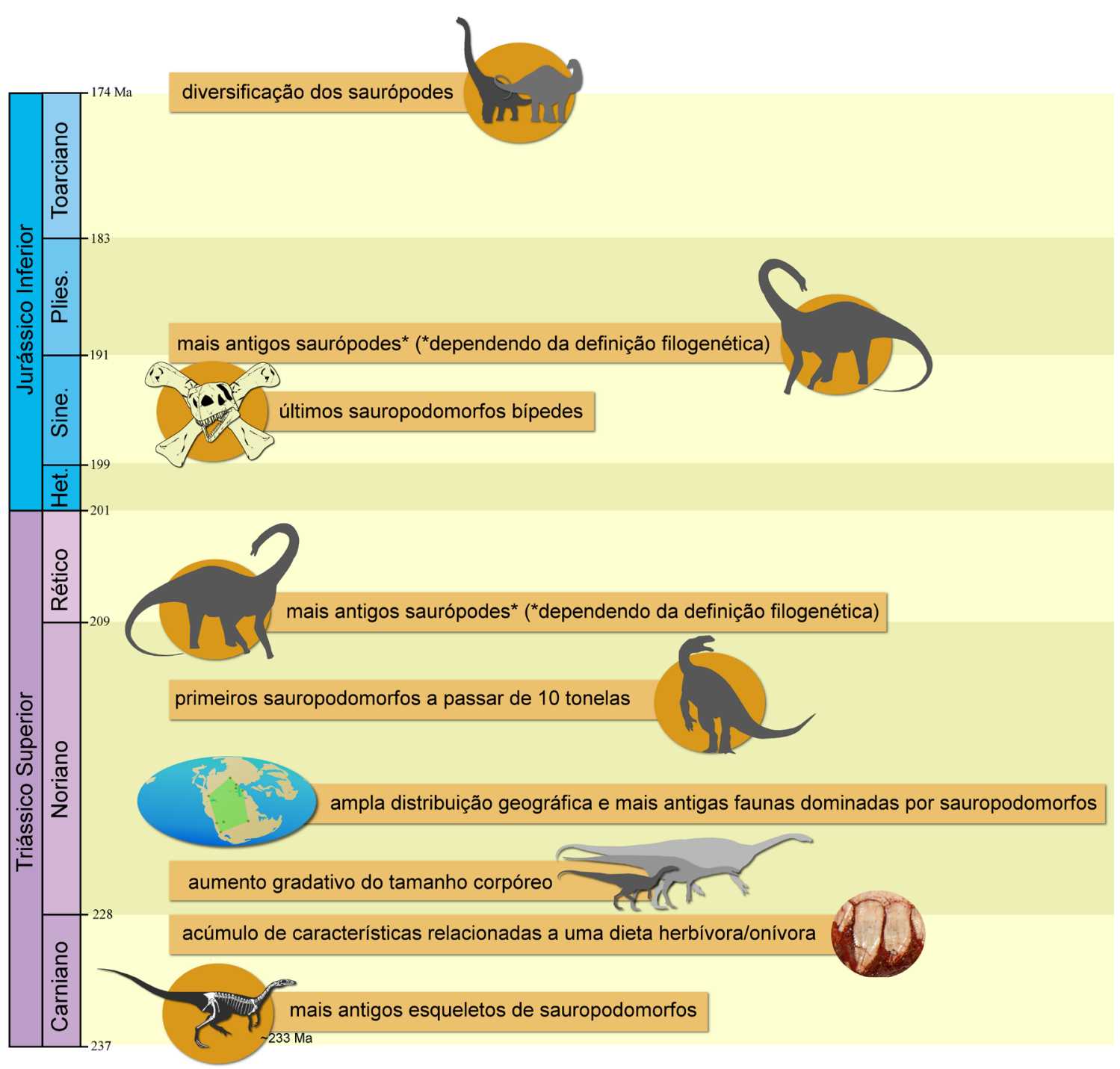

Figura 7. Padrões macroevolutivos dos sauropodomorfos ao longo do Triássico Superior e Jurássico Inferior

tetrápodes coevos não teriam acesso. Essa vantagem pode ter sido um dos principais gatilhos para o sucesso evolutivo do grupo durante o Noriano, momento em que os sauropodomorfos deixam de ocupar apenas o sudoeste do Pangea para alcançar uma distribuição cosmopolita (Müller et al., 2017).

Não só se observa um aumento claro da distribuição geográfica dos sauropodomorfos durante o Noriano, como também é evidente o aumento do número de espécimes coletados em sítios fossilíferos onde os mesmos ocorrem (e.g. Martínez et al., 2015). A mudança estaria relacionada com um aumento da abundância de sauropodomorfos nos ecossistemas terrestres, onde agora estes animais passariam a ser os herbívoros de grade porte de maior abundância em seus ambientes. Essa é a primeira vez na história em que os dinossauros exercem um papel de domínio nos ecossistemas.
Depois desse evento, os dinossauros passaram a reinar durante o restante da Era Mesozoica, a qual é conhecida até hoje como a idade dos répteis, mais especificamente dos famigerados 'Répteis Terríveis' de Owen. No entanto, os fósseis escavados em rochas da América do Sul nos mostram que alcançar o sucesso evolutivo não foi uma tarefa simples e não ocorreu de forma instantânea, mas sim, foi um processo longo e marcado por desafios, no qual os primeiros sauropodomorfos foram animais "tímidos" em um mundo dominado por répteis gigantes, vorazes e já bem estabelecidos.

\section{Referências}

Agnolín, F. L., \& Rozadilla, S. (2017). Phylogenetic reassessment of Pisanosaurus mertii Casamiquela, 1967, a basal dinosauriform from the Late Triassic of Argen-

\begin{tabular}{c|c|c|c|c|c}
\hline (C) Terrae Didat. & Campinas, SP & v.16 & $1-13$ & $\mathrm{e} 020013$ & 2020 \\
\hline
\end{tabular}


tina. Journal of Systematic Palaeontology, 16, 853-879. doi:10.1080/14772019.2017.1352623.

Apaldetti, C., Martínez, R. N., Cerda, I. A., Pol, D., \& Alcober, O. (2018). An early trend towards gigantism in Triassic sauropodomorph dinosaurs. Nature ecology \& evolution, 2(1227), 1-9. doi: 10.1038/s41559018-0599-y.

Apaldetti, C., Martinez, R. N., Pol, D., \& Souter, T. (2014). Redescription of the skull of Coloradisaurus brevis (Dinosauria, Sauropodomorpha) from the Late Triassic Los Colorados Formation of the Ischigualasto-Villa Union Basin, northwestern Argentina. Journal of Vertebrate Paleontology, 34, 11131132. doi: 10.1080/02724634.2014.859147.

Arcucci, A. (1986). Nuevos materiales y reinterpretacion de Lagerpeton chanarensis Romer (Thecodontia, Lagerpetonidae nov.) del Triasico Medio de La Rioja, Argentina. Ameghiniana, 23, 233-242.

Baron, M. G., Norman, D. B., \& Barrett, P. M. (2017). A new hypothesis of dinosaur relationships and early dinosaur evolution. Nature, 543, 501-506. doi: 10.1038/nature21700.

Barrett, P. M., Nesbitt, S. J., \& Peecook, B. R. (2015). A large-bodied silesaurid from the Lifua Member of the Manda beds (Middle Triassic) of Tanzania and its implications for body-size evolution in Dinosauromorpha. Gondwana Research, 27, 925-931. doi: 10.1016/j.gr.2013.12.015.

Benton, M. J. (1983). Dinosaur success in the Triassic: a noncompetitive ecological model. The Quarterly Review of Biology, 58, 29-55. doi: 10.1086/413056.

Benton, M. J. (1985). Classification and phylogeny of diapsid reptiles. Zoological Journal of the Linnean Society, 84, 97-164. doi: 10.1111/j.1096-3642.1985. tb01796.x.

Bittencourt, J. S., Da-Rosa, Á. A. S., Schultz, C.L., \& Langer, M. C. (2013). Dinosaur remains from the 'Botucaraí Hill' (Caturrita Formation), Late Triassic of south Brazil, and their stratigraphic context. Historical Biology, 25, 81-93. doi: 10.1080/08912963.2012.694881.

Bittencourt, J. S., Leal, L. A., Langer, M. C., \& Azevedo, S. A. K. (2012). An additional basal sauropodomorph specimen from the Upper Triassic Caturrita Formation, southern Brazil, with comments on the biogeography of plateosaurids. Alcheringa, 36, 269-278. doi: 10.1080/03115518.2012.634111.

Bronzati, M., Müller, R. T., \& Langer, M. C. (2019). Skull remains of the dinosaur Saturnalia tupiniquim (Late Triassic, Brazil): With comments on the early evolution of sauropodomorph feeding behaviour. PloS one, 14(9), e0221387. doi: 10.1371/journal. pone.0221387.

Bronzati, M., Rauhut, O. W., Bittencourt, J. S., \& Langer, M. C. (2017). Endocast of the Late Triassic (Carnian) dinosaur Saturnalia tupiniquim: implications for the evolution of brain tissue in Sauropodomorpha.
Scientific reports, 7 (11931), 1-7. doi: 10.1038/s41598017-11737-5.

Brusatte, S. L., Benton, M. J., Ruta, M., \& Lloyd, G. T. (2008). Superiority, competition, and opportunism in the evolutionary radiation of dinosaurs. Science, 321, 1485-1488. doi: 10.1126/science.1161833.

Brusatte, S. L., Niedzwiedzki, G., \& Butler, R. J. (2011). Footprints pull origin and diversification of dinosaur stem lineage deep into Early Triassic. Proceedings of the Royal Society of London, Biological Sciences, 278, 1107-1113. doi: 10.1098/rspb.2010.1746.

Cabreira, S. F., Kellner, A. W., Dias-da-Silva, S., Robertoda-Silva, L., Bronzati, M., Marsola, J. A. C., Müller, R. T., Bittencourt, J. S., Batista, B. J., Raugust, T., Carrilho, R., Brodt, A., \& Langer, M. C. (2016). A Unique Late Triassic Dinosauromorph Assemblage Reveals Dinosaur Ancestral Anatomy and Diet. Current Biology, 26, 3090-3095. doi: 10.1016/j. cub.2016.09.040.

Cabreira, S. F., Schultz, C. L., Bittencourt, J. S., Soares, M. B., Fortier, D. C., Silva, L. R., \& Langer, M. C. (2011). New stem-sauropodomorph (Dinosauria, Saurischia) from the Triassic of Brazil. Naturwissenschaften, 98, 1035-1040. doi: 10.1007/s00114-0110858-0.

Casamiquela, R. M. (1967). Un nuevo dinosaurio ornitischio Triásico (Pisanosaurus mertii, Ornithopoda) de la Formación Ischigualasto, Argentina. Ameghiniana, 4, 47-64.

Dzik, J. (2003). A beaked herbivorous archosaur with dinosaur affinities from the early Late Triassic of Poland. Journal of Vertebrate Paleontology, 23, 556-574. doi: 10.1671/A1097.

Ezcurra, M. D. (2012). Comments on the taxonomic diversity and paleobiogeography of the earliest known dinosaur assemblages (late Carnian - earliest Norian). Revista de Historia Natural, Nueva Serie, 2, 49-71.

Ezcurra, M.D. (2017). A new early coelophysoid neotheropod from the Late Triassic of Northwestern Argentina. Ameghiniana, 54, 506-538. doi: 10.5710/ AMGH.04.08.2017.3100.

Ferigolo, J., Langer, \& M. C. (2007). A Late Triassic dinosauriform from south Brazil and the origin of the ornithischian predentary bone. Historical Biology, 19, 23-33. doi: 10.1080/08912960600845767.

Galton, P. M., \& Upchurch, P. 2004. Prosauropoda. In: Weishampel, D. B., Dodson P., \& Osmólska H. eds. 2004. The Dinosauria. $2^{\text {nd }}$ ed. Berkeley: University of California Press. p. 232-258.

Garcia, M. S., Müller, R. T., Da-Rosa Á. A., \& Dias-daSilva S. (2019a). The oldest known co-occurrence of dinosaurs and their closest relatives: a new lagerpetid from a Carnian (Upper Triassic) bed of Brazil with implications for dinosauromorph biostratigraphy, early diversification and biogeography. Journal of South American Earth Sciences, 91, 302-319. doi: 10.1016/j.jsames.2019.02.005.

\begin{tabular}{c|c|c|c|c|c}
\hline (C) Terrae Didat. & Campinas, SP & v.16 & $1-13$ & $\mathrm{e} 020013$ & 2020 \\
\hline
\end{tabular}


Garcia, M. S., Pretto F. A., Dias-da-Silva S., \& Müller R. T. (2019b). A dinosaur ilium from the Late Triassic of Brazil with comments on key-character supporting Saturnaliinae. Anais da Academia Brasileira de Ciências, 91(Suppl. 2), e20180614. doi: 10.1590/00013765201920180614.

Griffin, C.T., \& Nesbitt, S. J. (2016). Anomalously high variation in postnatal development is ancestral for dinosaurs but lost in birds. Proceedings of the National Academy of Sciences, 113 (51), 14757-14762. doi: 10.1073/pnas.1613813113.

Horn, B. L. D., Melo, T. M., Schultz, C. L., Philipp, R. P., Kloss, H. P., \& Goldberg, K. (2014). A new third-order sequence stratigraphic framework applied to the Triassic of the Paraná Basin, Rio Grande do Sul, Brazil, based on structural, stratigraphic and paleontological data. Journal of South American Earth Sciences, 55, 123-132. doi: 10.1016/j.jsames.2014.07.007.

Irmis, R. B., Nesbitt, S. J., Padian, K., Smith, N. D., Turner, A. H., Woody, D., \& Downs, A. (2007). A Late Triassic dinosauromorph assemblage from New Mexico and the rise of dinosaurs. Science, 317, 358-361. doi: 10.1126/science.1143325.

Langer, M. C., \& Ferigolo, J. (2013). The Late Triassic dinosauromorph Sacisaurus agudoensis (Caturrita Formation, Rio Grande do Sul, Brazil): anatomy and affinities. Geological Society London Special Publications, 379, 353-392. doi: 10.1144/SP379.16.

Langer, M. C., Abdala, F, Richter, M., \& Benton, M. J. (1999). A sauropodomorph dinosaur from the Upper Triassic (Carnian) of Southern Brazil. Acad Sci Paris Sci Terre et Planetnét, 329, 511-517.

Langer, M. C., Ezcurra, M. D., Bittencourt, J. S., \& Novas, F. E. (2010). The origin and early evolution of dinosaurs. Biological Reviews, 85, 55-110. doi: 10.1111/j.1469-185X.2009.00094.x.

Langer, M. C., Ramezani, J., \& Da-Rosa, Á. A. (2018). $\mathrm{U}-\mathrm{Pb}$ age constraints on dinosaur rise from south Brazil. Gondwana Research, 57, 133-140. doi: 10.1016/j.gr.2018.01.005.

Leal, L. A., Azevedo, S. A. K., Kellner, A. W., \& Da-Rosa, Á. A. S. (2004). A new early dinosaur (Sauropodomorpha) from the Caturrita Formation (Late Triassic), Paraná Basin, Brazil. Zootaxa, 690, 1-24. doi: 10.5281/zenodo.169421.

Marsola, J. C., Bittencourt, J. S., Butler, R. J., Da-Rosa, Á. A. S., Sayão, J. M., \& Langer, M. C. 2018. A new dinosaur with theropod affinities from the Late Triassic Santa Maria Formation, South Brazil. Journal of Vertebrate Paleontology, 38 (5), e1531878. doi: 10.1080/02724634.2018.1531878.

Martínez, R. N., \& Apaldetti, C. (2017). A Late Norian-Rhaetian Coelophysid Neotheropod (Dinosauria, Saurischia) from the Quebrada Del Barro Formation, Northwestern Argentina. Ameghiniana, 54, 488-505. doi: 10.5710/AMGH.09.04.2017.3065.

Martínez, R. N., Apaldetti, C., Alcober, O. A., Colombi,
C. E., Sereno, P. C., Fernandez, E., Santi Malnis, P., Correa, G. A, \& Abelín, D. (2013). Vertebrate succession in the Ischigualasto Formation. Journal of Vertebrate Paleontology, 31, 10-30. doi: 10.5710/ AMGH.09.04.2017.3065.

Martínez, R. N., Apaldetti, C., Correa, G. A., \& Abelín, D. (2016). A Norian lagerpetid dinosauromorph from the Quebrada del Barro Formation, northwestern Argentina. Ameghiniana, 53, 1-13. doi: 10.5710/AMGH.21.06.2015.2894.

Martínez, R. N., Apaldetti, C., Correa, G., Colombi, C. E., Fernández, E., Malnis, P. S., Padrerio, A., Abelín, D., Benegas, L. G., Aguilar-Cameo, A., \& Alcober, O. A. (2015). A new Late Triassic vertebrate assemblage from northwestern Argentina. Ameghiniana, 52 (4), 379-391. doi: 10.5710/AMGH.27.04.2015.2889.

Martínez, R. N., Sereno, P. C., Alcober, O. A., Colombi, C. E., Renne, P. R., Montañez, I. P, \& Currie, B. S. (2011). A basal dinosaur from the dawn of the dinosaur era in the western Pangaea. Science, 331, 206210. doi: 10.1126/science.1198467.

Mcphee, B. W., \& Choiniere, J. N. (2017). The osteology of Pulanesaura eocollum: implications for the inclusivity of Sauropoda (Dinosauria). Zoological Journal of the Linnean Society, 182, 830-861. doi: 10.1093/zoolinnean/zlx074.

Mcphee, B. W., Benson, R. B., Botha-Brink, J., Bordy, E. M., \& Choiniere, J. N. (2018). A giant dinosaur from the earliest Jurassic of South Africa and the transition to quadrupedality in early sauropodomorphs. Current Biology, 28, 3143-3151. doi: 10.1016/j.cub.2018.07.063.

Müller, R.T. (2019). Craniomandibular osteology of Macrocollum itaquii (Dinosauria: Sauropodomorpha) from the Late Triassic of Southern Brazil. Journal of Systematic Palaeontology, Online First. doi: 10.1080/14772019.2019.1683902.

Müller, R. T., Da-Rosa, Á. A. S., Silva, L. R., Aires, A. S. S., Pacheco, C. P., Pavanatto, A. E. B., \& Dias-daSilva, S. (2015). Wachholz, a new exquisite dinosaur bearing fossiliferous site from the Upper Triassic of southern Brazil. Journal of South American Earth Sciences, 62, 120-128. doi: 10.1016/j.jsames.2014.10.009.

Müller, R. T., \& Garcia, M. S. (2019). Rise of an empire: analysing the high diversity of the earliest sauropodomorph dinosaurs through distinct hypotheses. Historical Biology, Online First, 1-6. doi: 10.1080/08912963.2019.1587754.

Müller, R. T., Langer, M. C., \& Dias-da-Silva, S. (2017). Biostratigraphic significance of a new early sauropodomorph specimen from the Upper Triassic of southern Brazil. Historical Biology, 29, 187-202. doi: 10.1080/08912963.2016.1144749.

Müller, R. T., Langer, M. C., \& Dias-Da-Silva, S. (2018a). Ingroup relationships of Lagerpetidae (Avemetatarsalia: Dinosauromorpha): a further phylogenetic investigation on the understanding of dinosaur

\begin{tabular}{c|c|c|c|c|c}
\hline (C) Terrae Didat. & Campinas, SP & v.16 & $1-13$ & $\mathrm{e} 020013$ & 2020 \\
\hline
\end{tabular}


relatives. Zootaxa, 4392, 149-158. doi: 10.11646/ zootaxa.4392.1.7.

Müller, R. T., Langer, M. C., \& Dias-da-Silva, S. (2018c). An exceptionally preserved association of complete dinosaur skeletons reveals the oldest long-necked sauropodomorphs. Biology letters, 14 (11), 20180633. doi: 10.1098/rsbl.2018.0633.

Müller, R. T., Langer, M. C., Bronzati, M., Pacheco, C. P., Cabreira, S. F., \& Dias-da-Silva, S. (2018b). Early evolution of sauropodomorphs: anatomy and phylogenetic relationships of a remarkably well-preserved dinosaur from the Upper Triassic of southern Brazil. Zoological Journal of the Linnean Society, 184, 1187-1248. doi: 10.1093/zoolinnean/zly009.

Müller, R. T., Langer, M. C., Pacheco, C. P., \& Dias-da-Silva, S. (2018d). The role of ontogeny on character polarization in early dinosaurs: a new specimen from the Late Triassic of southern Brazil and its implications. Historical Biology, 31, 794-805. doi: 10.1080/08912963.2017.1395421.

Nesbitt, S. J., Butler, R. J., Ezcurra, M. D., Barrett, P. M., Stocker, M. R., Angielczyk, K. D., Smith, R. M. H., Sidor, C. A., Niedzwiedzki, G., Sennikov, A. G., \& Charing, A. J. (2017). The earliest bird-line archosaurs and the assembly of the dinosaur body plan. Nature, 544, 484-487. doi: 10.1038/nature22037.

Nesbitt, S. J., Irmis, R. B., Parker, W. G., Smith, N. D., Turner, A. H., \& Rowe, T. (2009). Hindlimb osteology and distribution of basal dinosauromorphs from the Late Triassic of North America. Journal of Vertebrate Paleontology, 29, 498-516. doi: 10.1671/039.029.0218.

Nesbitt, S. J., Sidor, C. A., Irmis, R. B., Angielczyk, K. D., Smith, R. M. H., \& Tsuji, L. A. (2010). Ecologically distinct dinosaurian sister-group shows early diversification of Ornithodira. Nature, 464, 95-98. doi: 10.1038/nature08718.

Novas, F. E. (1992). La evolucioón de los dinosaurios carnívoros. In: Sanz, J.L., Buscalioni, A. eds. 1992. Los dinosaurios y su entorno biotico. Espanha: Actas II Curso de Paleontologíca en Cuenca. p. 125-163.

Owen, R. Report on British fossil reptiles, Part II. (1842). Reports of the British Association for the Advancement of Science, 11th Meeting, 60-204.

Pacheco, C. P., Müller, R. T., Langer, M. C., Pretto, F. A., Kerber, L., \& Dias-da-Silva, S. (2019). Gnathovorax cabreirai: a new early dinosaur and the origin and initial radiation of predatory dinosaurs. PeerJ, Online First. doi: 10.7717/peerj.7963.

Parrish, J. M. (1998). The origin of high browsing: the paleoecological significance of the sauropodomorph bauplan. Journal of Vertebrate Paleontology, 18.

Pinheiro, F. L. (2017). A fragmentary dinosaur femur and the presence of Neotheropoda in the upper Triassic of Brazil. Revista Brasileira de Paleontologia, 19, 211-
216. doi: 10.4072/rbp.2016.2.05

Pretto, F. A., Langer, M. C., \& Schultz, C. L. (2018). A new dinosaur (Saurischia: Sauropodomorpha) from the Late Triassic of Brazil provides insights on the evolution of sauropodomorph body plan. Zoological Journal of the Linnean Society, 185, 388-416. doi: 10.1093/zoolinnean/zly028.

Romer, A. S. (1971). The Chañares (Argentina) Triassic reptile fauna. $\mathrm{X}$. Two new but incompletely known long-limbed pseudosuchians. Breviora, 378, 1-10.

Rowe, T. B., Sues, H-D., \& Reisz, R. R. (2010). Dispersal and diversity in the earliest North American sauropodomorph dinosaurs, with a description of a new taxon. Proceedings of the Royal Society B: Biological Sciences, 278, 1044-1053. doi: 10.1098/rspb.2010.1867 .

Salgado, L., Coria, R. A., \& Calvo, J. O. (1997). Evolution of titanosaurid sauropods. I. Phylogenetic analysis based on the postcranial evidence. Ameghiniana, 34, 3-32.

Sarigül, V. (2016). New basal dinosauromorph records from the Dockum Group of Texas, USA. Palaeontologia Electronica, 19, 1-13. doi: 10.26879/564.

Seeley, H. G. (1887). On the classification of the fossil animals commonly named Dinosauria. Proceedings of the Royal Society of London, 43, 165-171.

Sereno, P. C., Forster, C. A., Rogers, R. R., \& Monetta, A. M. (1993). Primitive dinosaur skeleton from Argentina and the early evolution of the Dinosauria. Nature, 361, 64-66.

Sertich, J. J., \& Loewen, M. A. (2010). A new basal sauropodomorph dinosaur from the Lower Jurassic Navajo Sandstone of southern Utah. PLoS One, 5 (3e9789), 1-17. doi: 10.1371/journal.pone.0009789.

Taylor, M. P., Upchurch, P., Yates, A. M., Wedel, M. J., \& Naish, D. (2010). Sauropoda. In: De Queiroz, K., Cantino, P. D., Gauthier, J. A. eds. 2010. Phylonyms: a Companion to the PhyloCode. Berkley: University of California Press.

Upchurch, P., Barrett, P. M., \& Galton, P. M. (2007). A phylogenetic analysis of basal Sauropodomorph relationships: implications for the origin of sauropod dinosaurs. Special Papers in Palaeontology, 77, 57-90.

Yates, A. M. (2007). The first complete skull of the Triassic dinosaur Melanorosaurus Haughton (Sauropodomorpha: Anchisauria). Special Papers in Palaeontology, 77, 9-5.

Zerfass, H., Lavina, E. L., Schultz, C. L., Garcia, A. J. V., Faccini, U. F., \& Chemale, Jr F. (2003). Sequence stratigraphy of continental Triassic strata of Southernmost Brazil: a contribution to Southwestern Gondwana palaeogeography and palaeoclimate. Sedimentary Geology, 161, 85-105. doi: 10.1016/ S0037-0738(02)00397-4. 